DOSSIER

\title{
LUCIE BÉGIN
}

EM Normandie, Métis

DIDIER CHABAUD

Université de Cergy; EM Normandie

\section{La résilience des organisations \\ Le cas d'une entreprise familiale}

\begin{abstract}
La résilience organisationnelle renvoie à la capacité de rebondir face à l'inattendu. Nous proposons d'opérationnaliser ce concept autour de trois dimensions qui distinguent la capacité : d'absorption du choc, de renouvellement et d'appropriation. L'étude du cas d'une entreprise familiale fondée en 1826, qui a été confrontée à trois chocs majeurs au cours de son existence, permet de montrer la façon dont la résilience organisationnelle se déploie et se construit pour permettre sa survie.
\end{abstract}


$\mathrm{L}$ a durée de vie des entreprises familiales (EF) interroge. Selon l'aphorisme populaire, la longévité d'une $\mathrm{EF}$ ne dépasse généralement pas les trois générations: la première fonde l'entreprise, la seconde la consolide et la troisième l'enterre, funeste destin appelé l'effet Buddenbrooks ${ }^{1}$. De fait, selon Ward (1987), moins de $10 \%$ des EF atteignent la troisième génération ce qui n'empêche pas de trouver des exemples impressionnants de longévité, comme c'est le cas pour les 41 bicentenaires de l'Association des Hénokiens.

Pourtant, au-delà de leur longévité, leurs histoires sont souvent parsemées de difficultés et de revers de fortune. Ainsi que le soulignent Hamel et Välikangas «Au lieu de voler de succès en succès, la plupart des firmes vont de succès en échec et, après un long et dur chemin, connaissent à nouveau le succès. La résilience se réfère à une capacité de reconstruction continuelle. » (2003, p. 6). Elle permet de cerner pourquoi, face aux chocs et événements inattendus ou soudains qu'elles rencontrent dans leur parcours, certaines entreprises sont capables de rebondir alors que d'autres s'effondrent et disparaissent (Weick et Suncliffe, 2007; Christianson et al., 2009).

C'est en étudiant le choc ou l'inattendu, vu comme une discontinuité par rapport au cours normal des activités ${ }^{2}$, que nous abordons la résilience organisationnelle. Pour ce faire, nous analysons le cas ERD et $\mathrm{C}^{\mathrm{ie}}$, une $\mathrm{EF}$ fondée en 1826, aujourd'hui dans les mains de la sixième génération, dont plus de $95 \%$ du capital est détenu par la famille Raoul-Duval (RD). Depuis l'origine, son activité principale a été le négoce, métier d'intermédiaire « où, continuellement, vendeurs et acheteurs cherchent à se passer de nos services en traitant directement de l'origine à la consommation ${ }^{3} »$. Dans ce métier où l'avantage concurrentiel est hautement volatil - et encore plus, dans le cas du négoce sur des produits exotiques ou provenant de pays à risques -, ERD et $\mathrm{C}^{\text {ie }} \mathrm{a}$ connu des chocs majeurs qui ont ébranlé sa structure et ses sources de revenus. Face à ces revers de fortune, elle a été obligée de rebondir, en renouvelant ses marchés et ses produits, voire en repensant son métier, pour survivre dans la durée.

Après une revue de la littérature qui permet de clarifier, et d'opérationnaliser, la notion de résilience organisationnelle, nous relatons l'histoire de ERD et $\mathrm{C}^{\mathrm{ie}}$ afin de mettre en évidence les chocs qui l'ont émaillée et d'analyser les pratiques qui lui ont permis de faire face et de rebondir. La discussion permet alors de souligner la portée de la résilience dans l'EF, et d'analyser les sources.

\section{I - LA RÉSILIENCE ORGANISATIONNELLE}

Après avoir retracé la façon dont le concept de résilience a été transposé des individus aux organisations, nous en proposons l'opérationnalisation, autour de trois dimensions.

1. L'effet Buddenbrooks renvoie au livre homonyme de Thomas Mann, paru en 1901, lequel raconte le déclin d'une entreprise familiale dont les héritiers dilapident le patrimoine.

2. Voir Altintas et Royer (2009) pour une discussion approfondie du concept et de sa connexion aux turbulences de l'environnement.

3. Document interne (correspondance d'affaires), 24 février 1958. 


\section{La résilience: d'une approche individuelle à un éclairage organisationnel}

L'idée de résilience n'est pas nouvelle. Dans la médecine traditionnelle chinoise, elle désigne la capacité des systèmes vivants de se régénérer après une blessure grave. En physique, elle renvoie à la capacité interne d'un matériau à retrouver sa forme initiale après avoir reçu un choc. En psychologie, elle désigne la capacité de l'individu à résister aux traumatismes, à rebondir face à l'adversité, à retomber sur ses pieds malgré les déboires de sa vie. Dans cette perspective, Boris Cyrulnik ${ }^{4}$ définit la résilience comme une qualité intrinsèque de l'individu, qui lui permet de dépasser sa souffrance, d'apprendre de ses expériences douloureuses et de devenir plus fort. S'inscrivant dans cette école de pensée, Bernard (2007) voit dans l'acte d'entreprendre un processus de reconstruction de soi, d'affirmation et d'indépendance, voire de revanche face aux mauvais coups de la vie, ce qu'elle appelle la résilience entrepreneuriale.

Dans les sciences de gestion, le concept de résilience organisationnelle a d'abord fait son apparition dans le cadre des recherches sur la gestion des crises et les organisations à haute fiabilité. L'analyse passe alors du niveau individuel au niveau organisationnel et collectif, sur « les mécanismes qui rendent le groupe moins vulnérable aux ruptures du sensemaking " (Weick, 1993, p. 628), voire sur la capacité qu'aura la firme à absorber, à répondre et à capitaliser sur des perturbations issues de change- ments de l'environnement (Lengnick-Hall et Beck, 2005, 2009). Ce que recouvre le concept demeure cependant sujet à débat dans la mesure où différentes définitions existent et qu'il est difficile de mesurer $a$ priori la capacité de résilience organisationnelle, même si des efforts ont été faits en ce sens (Somers, 2009). Par contre, l'étude des réactions et actions d'entreprises ayant survécu à des chocs ou événements inattendus met en lumière les mécanismes qui fondent la capacité de résilience (Lengnick-Hall et Beck, 2005; Weick et Sutcliffe, 2007). Elle conduit à une analyse attentive des facteurs et des processus qui permettent à l'entreprise de traverser les épreuves et d'en ressortir éventuellement plus forte et invite à se pencher la manière dont l'organisation construit et entretient cette capacité de résilience dans le temps (Hollganel et al., 2009).

\section{Les dimensions de la résilience organisationnelle}

Selon Coutu (2002), les entreprises résilientes se caractérisent par trois aspects: 1) elles sont pragmatiques et font face à la réalité, sans faire montre d'un optimisme démesuré ; 2) elles ont un fort système de valeurs partagées qui permet de donner du sens aux difficultés ou défis rencontrés; 3) elles sont ingénieuses en ce qu'elles savent tirer parti de leurs ressources pour bricoler des solutions nouvelles face aux situations inhabituelles qu'elles rencontrent. Pour Hamel et Välikangas (2003), les entreprises résilientes doivent faire face à quatre défis: 1) un défi cognitif, car elles doivent être réa-

4. Boris Cyrulnik a publié de nombreux ouvrages sur la résilience dont Un merveilleux malheur (2002), Les vilains petits canards (2004), Le murmure des fantômes (2005), Je me souviens (2009). 
listes face aux changements, et conscientes qu'ils vont affecter l'organisation, 2) un défi stratégique qui requiert d'avoir la capacité à imaginer de nouvelles options stratégiques face à la stratégie déclinante, 3) un défi politique qui demande de réallouer les ressources de manière à supporter les activités prometteuses pour le futur et à abandonner les produits et programmes du passé, 4) un défi idéologique qui consiste à insuffler une attitude proactive et axée sur la recherche continuelle des nouvelles opportunités.

La résilience suppose ainsi de combiner à la fois une approche défensive - tenant aux mesures de précaution et de gestion du risque en aval qui permettent de faire face au choc lorsqu'il se produit - et une approche proactive - être ingénieux et créatif pour imaginer des solutions nouvelles et prendre des actions lui permettant de se régénérer. Mais l'entreprise doit, au-delà, pouvoir apprendre des crises traversées (autoréflexion) pour être mieux armée face à l'avenir; elle doit profiter de la discontinuité créée par le choc pour identifier ses faiblesses et les corriger (Christianson et al., 2009). La résilience suppose donc trois dimensions fortement imbriquées les unes aux autres: 1) une capacité d'absorption, permettant à l'entreprise de ne pas s'effondrer face à l'inattendu ou au choc ; 2) une capacité de renouvellement par laquelle elle peut s'inventer de nouveaux futurs ; 3 ) une capacité d'appropriation lui permettant de devenir plus forte de ses expériences (Weick et Sutcliffe, 2007).

\section{La capacité d'absorption}

La capacité d'absorption suppose que l'entreprise puisse faire face aux chocs tout en évitant l'effondrement, ce qui nécessite non seulement l'existence de moyens/ressources mais aussi une volonté de continuité chez les dirigeants.

Pour résister aux chocs et survivre aux conséquences qui en découlent, l'entreprise doit pouvoir mobiliser des ressources, que celles-ci soient immédiatement disponibles sous la forme d'un excédent organisationnel (organizational slack) (Cyert et March, 1963) ou potentiellement mobilisables auprès de sources externes (soutien, prêt, assistance, alliance, etc.). La présence d'un excédent organisationnel permet de protéger la firme des turbulences de l'environnement, mais aussi de favoriser l'innovation en donnant la capacité de redéployer des ressources en fonction de besoins (De Carolis et al., 2009). Dans le cas d'une EF, cet aspect peut signifier de pouvoir prendre appui sur la famille ${ }^{5}$, et plus largement sur le capital familial qu'il soit financier, social ou humain (Arregle et al., 2007; Danes et al., 2009). En ce sens, la résilience rend possible la continuité : être une entreprise qui a engrangé des résultats positifs sur plusieurs années - en constituant des réserves - permet de financer un plan de restructuration ou de faire face à une brusque dégradation des résultats sans être acculé à la faillite. Il en va de même des ressources potentielles telles que la bonne réputation d'une entreprise qui lui permet d'obtenir des « emprunts exceptionnels » et

5. De Carolis et al. (2009) montrent ainsi comment l'organizational slack renvoie notamment aux ressources que l'entreprise est en mesure de lever auprès de financeurs externes en cas de choc. 
des partenaires loyaux qui peuvent donner un coup de pouce lorsque requis. Dans le même ordre d'idée, l'adossement à une famille soudée apparaît comme une ressource organisationnelle précieuse face aux difficultés.

Mais il ne suffit pas de pouvoir, encore faut-il vouloir continuer l'aventure organisationnelle. En effet, la volonté de s'en sortir des dirigeants est essentielle car c'est à eux que revient la décision de mobiliser les ressources pour financer la continuité et le renouvellement. Ici, l'EF peut être avantagée, en bénéficiant de l'affectio societatis des membres de la famille, qu'ils travaillent ou non dans celle-ci. Par désir dynastique, par tradition ou par attachement, ces derniers peuvent être tentés de mobiliser des ressources pour assurer la survie de l'EF, alors même qu'une solution « économiquement rationnelle » pourrait pousser à liquider la société.

\section{La capacité de renouvellement}

Au-delà de la capacité à résister, l'entreprise doit être capable d'agir et d'imaginer des solutions inédites face aux situations inhabituelles (Lengnick-Hall et Beck, 2005 et 2009; Hamel et Välikangas, 2003). C'est ce que nous appelons la «capacité de renouvellement », par laquelle l'entreprise cherche à développer de nouvelles activités et/ou à repenser les activités existantes ou en expérimentant de nouvelles façons de faire. Cette capacité rejoint les travaux sur l'orientation entrepreneuriale de la firme (Miller, 1983; Stevenson et Jarillo, 1990) et sur les processus de régénération stratégique (Stopford et Baden-Fuller, 1994), ce qui permet de mettre l'accent sur quelques facteurs clés: 1) la proactivité dans la recherche des opportunités, 2) des aspirations qui vont audelà de leurs capacités actuelles, 3) la mobilisation de l'équipe de direction et de management. Ces éléments s'accompagnent d'une attention forte à l'utilisation au mieux des ressources disponibles et de la limitation de la prise de risque, compte tenu du contexte « tendu » de l'entreprise.

\section{La capacité d'appropriation}

Pour être résiliente, l'organisation doit pouvoir tirer des leçons des chocs auxquels elle a dû faire face afin d'en sortir grandie, d'apprendre par elle-même (Christianson et $a l ., 2009)$. Thorne souligne que « tirer parti de ses échecs signifie de reconnaître leur contribution à l'apprentissage plutôt que d'ignorer ou nier ceux-ci » (2000, p. 313). La prise de conscience de la crise et de ses impacts est essentielle pour remettre en perspective les pratiques et routines: il est alors possible de réaliser un « apprentissage post-crise » qui permettra à l'entreprise de mieux préparer l'avenir (Altintas et Royer, 2009).

Cependant, cette troisième dimension de la capacité de résilience, demeure difficile à observer. D'une part, l'apprentissage nécessite du temps de réflexion et une prise de distance que les dirigeants n'ont souvent pas le loisir de s'accorder lorsqu'ils sont dans la tourmente d'un choc déstabilisateur, qu'ils sont dans l'urgence de décider et d'agir (Weick, 1993). C'est dire que le chercheur n'aura que rarement l'occasion d'assister en direct à l'émergence des apprentissages qui se font aussi au gré des discussions - dans la famille ou dans l'entreprise - autour des chocs et actions à prendre ou prises pour assurer la continuité de l'entreprise. Certaines déci- 
sions pourront être jugées a posteriori bonnes ou mauvaises, mais dans tous les cas pour en tirer les leçons, il faudra laisser retomber la poussière sur les événements avant de les décortiquer. D'autre part, les expériences vécues sont ellesmêmes transformées et réinterprétées avant d'être réintégrées et reprises dans les narrations que font les acteurs de ce qu'ils ont vécu. Ainsi se construit la mémoire organisationnelle qui sera transmise et réifiée avec le passage du temps et dans laquelle s'inscriront les apprentissages eux-mêmes non observables. Il faut donc chercher dans les histoires reprises et racontées au fil du temps pour trouver la sédimentation des événements passés inscrite dans les principes de gestion et la culture qui fondent la manière d'opérer dans l'entreprise (Giddens, 1987). Il y aura donc toujours un décalage dans le temps entre le moment où une crise intervient et celui où de nouvelles représentations du monde, voire de nouvelles routines, verront jour suite au processus d'apprentissage et d'autoréflexion.

La capacité d'appropriation des expériences passées, aussi douloureuses soientelles dans la mémoire organisationnelle, accroît la capacité de résilience de l'entreprise. L'avantage des entreprises familiales tient ici dans leur capacité à transmettre les fruits de ces apprentissages d'une génération à l'autre (Smith, 2009).

Dans la prochaine section, en nous appuyant sur le cas de l'entreprise familiale ERD et $\mathrm{C}^{\mathrm{ie}}$, nous allons décrire trois chocs majeurs auxquels les dirigeants ont été confrontés afin de faire ressortir les actions prises pour y faire face et rebondir.

\section{MÉTHODOLOGIE}

D'un point de vue méthodologique, le recours à un cas unique est une approche relativement fréquente dans les travaux sur les entreprises familiales, et plus généralement en gestion (Hlady-Rispal, 2002; David, 2003). Bien qu'elle limite la possibilité de généralisation des résultats et est parfois jugée anecdotique, l'étude de cas unique offre l'avantage de permettre une analyse en profondeur de l'entreprise étudiée et de prendre en compte le contexte dans lequel son histoire se déroule (Yin, 2003).

Pour réaliser le cas ERD et Cie, nous avons mené plus de quinze entretiens entre 2007 et 2009, auprès des membres de la direction et d'une employée de longue date. À ces données de première main, se sont ajoutées les recherches dans les archives de l'entreprise composées des procès-verbaux des assemblées générales (AG), des réunions du conseil d'administration (CA) de ERD et $\mathrm{C}^{\text {ie }}$ et de ses filiales, depuis 1932 jusqu'à nos jours, des livres de comptes et de diverses notes confidentielles et rapports internes. Une note sur l'histoire du groupe familial ERD et Cie, rédigée par Mathieu Goguel en 2005 a complété ces sources internes. L'étude du contexte s'est appuyée sur les travaux d'historiens tels Claude Malon sur Le Havre colonial (2006) et Hubert Bonin (2008) sur l'histoire du commerce outre-mer de 1887 à 2007, et la thèse de Nathalie Aubourg (2000) sur le négoce du café au Havre. 


\section{II - LE CAS ERD ET CIE : CRISES ET REBONDS}

Fondée en 1826 au Havre, ERD et $C^{\text {ie }}$ compte aujourd'hui environ 70 employés qui se répartissent dans deux activités principales : la production de produits chimiques et le négoce du bois. Sur près de deux siècles d'existence, ERD et $\mathrm{C}^{\mathrm{ie}}$ a dû faire face aux évolutions normales de ses environnements (arrivée du bateau vapeur, industrialisation de processus, développement des communications, etc.) et s'y adapter. Elle a aussi été confrontée à des chocs majeurs, imprévus et soudains (coup d'État, nationalisation, effondrement de marchés), qui ont parfois menacé sa survie et causé la perte d'investissements importants. Pourtant, l'entreprise est toujours là alors que d'autres ont cessé toute activité. Preuve de sa capacité à résister aux aléas d'un métier très volatil, elle a notamment survécu à l'entre-deux guerres alors que, sur les quelques 250 entreprises de négoce que comptait alors la seule place havraise, près de deux tiers mettaient fin à leurs activités (Aubourg, 2000). Sans relater toute la saga de cette entreprise familiale, nous nous concentrons ici sur trois épisodes marquants survenus entre 19502009, qui nous permettent par la suite d'analyser les mécanismes sous-jacents à la résilience. Le tableau 1 présente une vue synoptique de cette période, en indiquant les actions prises pour se redéployer sur de nouveaux marchés (représentées par les étoiles) lorsque les activités courantes étaient perturbées par des crises, coups de tonnerre annonciateurs de perturbations imminentes.

\section{La SHIC, dans la tourmente de la décolonisation française}

Fondée en 1926 à Saigon (Vietnam), la Société havraise indochinoise de commerce (SHIC), avait pour principale fonction d'assurer les approvisionnements de la maisonmère (riz, poivre, kapok, peaux de serpents, etc.), d'éliminer une partie des intermédiaires locaux en négociant directement sur place et de vérifier sur place la qualité des produits avant leur exportation vers la France ou ailleurs dans le monde. Très prospère, la SHIC est organisée comme un " centre de profits », avec ses propres crédits et ses dirigeants sur place lesquels disposent d'une large autonomie afin de pouvoir être réactifs lorsque des opportunités se présentent. Mais la guerre d'Indochine vient modifier la donne.

Tout d'abord, « l'attaque dont a été l'objet, de la part des rebelles, les magasins loués par la Société à Cholou »... « a entraîné la destruction presque totale desdits magasins et des marchandises qui s'y trouvaient » (CA 104e séance, 8/10/1949). Au-delà de cet épisode, marginal financièrement, la question est posée de la politique de l'entreprise. Si « une politique d'extrême prudence » est adoptée (CA 104 ${ }^{\mathrm{e}}$ ), décision est prise de poursuivre l'activité, qui « représente pour nous une participation financière importante, mais surtout notre meilleur outil de travail » $\left(\mathrm{CA} 115^{\mathrm{e}}\right.$ séance, 2/9/1950). Dès lors, la société cherche à limiter ses risques tout en gérant au mieux son activité en Indochine. Très rapidement, elle utilise une partie de ses fonds au financement d'autres activités (dès 1948, soutien du développement d'activités en Afrique, 
puis financement d'une filiale africaine en 1952). Dans le même temps, elle développe une société à Phnom-Penh (1953) pour être moins dépendante du Vietnam, avant de rapatrier le siège social au Havre (1955), à la fin de la guerre d'Indochine. Par la suite, le bureau de Saigon sera fermé (1960), tout en cherchant à maintenir, par contre, une activité dans le pays: "ce que nous recherchons, par le canal de la SHIC, c'est à conserver des correspondants valables pour l'avenir, en nous libérant du très gros souci que représente la gestion d'une affaire de ce genre, à distance » (CA, 193 ééance, 1960). On le voit, plutôt que de s'accrocher à une activité qui devenait de plus en plus risquée quoique fort rémunératrice, les dirigeants cherchent immédiatement à se redéployer ailleurs pour qu'il n'y ait pas de rupture des activités, au plan global du groupe.

\section{La SHAC, les hauts et les bas de l'épopée ivoirienne}

L'aventure de la maison ERD et $\mathrm{C}^{\text {ie }}$ en Afrique coloniale offre un autre exemple d'événement catastrophique et déstabilisateur. Dès 1938, les transactions en Côte d'Ivoire débutent via des agents exportateurs sur place à Abidjan. Mais, lorsque la situation en Indochine devient des plus préoccupantes, les dirigeants envisagent alors de renforcer leur activité sur la Côte d'Afrique, ce qui débouche en 1951 sur des projets d'implantation, et la création effective de la Société havraise africaine de commerce (1952).

La SHAC adopte des pratiques innovantes dans le négoce du café, prenant des participations dans les exploitations des petits producteurs et à partir de l'indépendance (1960), elle traite directement avec les coopératives au grand dam des grandes compagnies coloniales (Malon, 2006). Le bureau de la SHAC devient une véritable plateforme d'exportation internationale à partir de l'Afrique (outre le café et le cacao, elle exporte du riz, de l'huile de palme, du sucre, etc.). Les affaires sont si florissantes que de multiples filiales sont créées, souvent en partenariat, dans les domaines connexes du transit (Prodexport, un tiers des parts), du pesage avec la Société de Bascule de Côte d'Ivoire (SBCI), du stockage avec la Société commune de stockage de produits (SCSP), etc. En 1978, une filiale à $100 \%$ est créée pour le décorticage du café, la SHAD (Société havraise africaine de décorticage).

Cependant, le développement de la SHAC ne va pas sans inquiétudes. L'indépendance nationale s'accompagne d'une " ivoirisation » des sociétés, qui entraîne en 1970 l'entrée d'une société de droit ivoirien dans la SHAC, à hauteur de $51 \%$ du capital (98 CA de la SHAC, 1970). Ces actions sont transmises à une société d'État en 1974, puis c'est l'État lui-même qui prend possession de ces actions en 1982 (Goguel, 2005) avant de rompre, en 1987, les accords commerciaux passés avec la SHAC. Événement catastrophique à l'échelle du groupe familial qui tire alors la majeure partie de ses revenus de cette filiale! ERD et $\mathrm{C}^{\text {ie }}$ prend action en justice face à cette éviction malhonnête et, plusieurs années plus tard, elle obtiendra des compensations, mais la structure des activités du groupe n'en est pas moins fortement ébranlée. Pourtant, durant toute la période des années 1960, les dirigeants avaient sans succès cherché à diversifier leurs activités, tout en s'en tenant à leur métier de négociants, étant convaincus que: " [La] diversification n'a de chance de réussir que si on continue à faire ce qu'on sait faire. Alors que 
savons-nous faire? Nous savons acheter à l'étranger ou vendre à l'étranger un produit suivant des techniques particulières mais qui peuvent s'appliquer à beaucoup d'autres articles. [...] Notre spécificité, c'est le réflexe du commerce international, savoir s'adapter, savoir vendre le même produit au même prix à des clients de dix nationalités différentes, c'est-à-dire aborder le client différemment. Et cela, qu'on lui vende un balai-brosse ou du café. Par contre, devenir industriel quand on est négociant, on ne sait pas et on ne saura pas faire. $»^{6}$

C'est par le rachat d'une société d'import/export de matériel alimentaire, la Société nouvelle de réorganisation et de modernisation de l'industrie alimentaire (SNRMIA), basée à Strasbourg, que la phase de diversification débute en 1970. L'opération se révèle d'abord très fructueuse et tous les espoirs sont permis d'en faire un axe de croissance pour l'avenir, jusqu'à ce qu'un client majeur dénonce son contrat lequel représentait plus de $40 \%$ de l'activité. L'entreprise est donc fermée en 1983 sans entraîner une perte importante mais réduisant à néant les efforts de développement dans ce secteur. Parallèlement, d'autres opportunités de diversification sont explorées :

- 1971 : prise de participation de $50 \%$ dans la société Services et transports-armement (STA). Spécialisée dans le transport de produits contaminants, STA connaît une forte croissance, de sorte que les autres actionnaires fondateurs, les frères Poylo, décident de s'émanciper de ERD et $C^{\text {ie }}$, en lui rachetant ses parts en 1985 .
- 1983 : création de la Société commerciale Raoul-Duval et Delebarre (SCRDD), filiale à $75 \%$ de ERD et $\mathrm{C}^{\text {ie }}$, dont l'activité est l'import/export de bois, grumes et sillages. Devenue Havraidex, cette société existe encore aujourd'hui, et a fait l'objet ces dernières années de développements importants au Gabon.

- 1984 : rachat de $66 \%$ des parts de la Compagnie française des extraits (CFE), qui produit des extraits végétaux utilisés dans la tannerie, la cosmétologie et l'alimentaire animal. De faible dimension, cette activité est perçue à fort potentiel pour l'avenir et sa direction a été confiée en 1999 à François Raoul-Duval (sixième génération), l'un des fils de Hubert Raoul-Duval. Reposant sur la capacité à développer et formuler de nouveaux produits à partir d'extraits végétaux, cette activité suppose d'aller sur des territoires encore peu exploités ou difficilement accessibles. C'est ainsi qu'un site a été créé au Nicaragua en 2004, pour permettre un changement de dimension.

En somme, conscients de leur forte dépendance envers les activités de leur filiale ivoirienne, la SHAC, et ayant tiré les leçons de l'expérience indochinoise, les dirigeants du groupe familial cherchent activement à prendre des participations dans des secteurs autres que le café ou le cacao, et en dehors $\mathrm{du}$ négoce des produits exotiques qui avaient à l'époque coloniale assuré leur prospérité. Toutefois, ces tentatives furent pour la plupart incapables de restaurer l'ampleur des activités qui avaient prévalu du temps de la SHIC ou de la SHAC,

6. Conférence de Hubert Raoul-Duval (alors P-DG) à l'université du Havre, le 30 novembre 1987 (p. 19). 
Tableau 1 - Vue synoptique des événements majeurs de la période 1950-2009

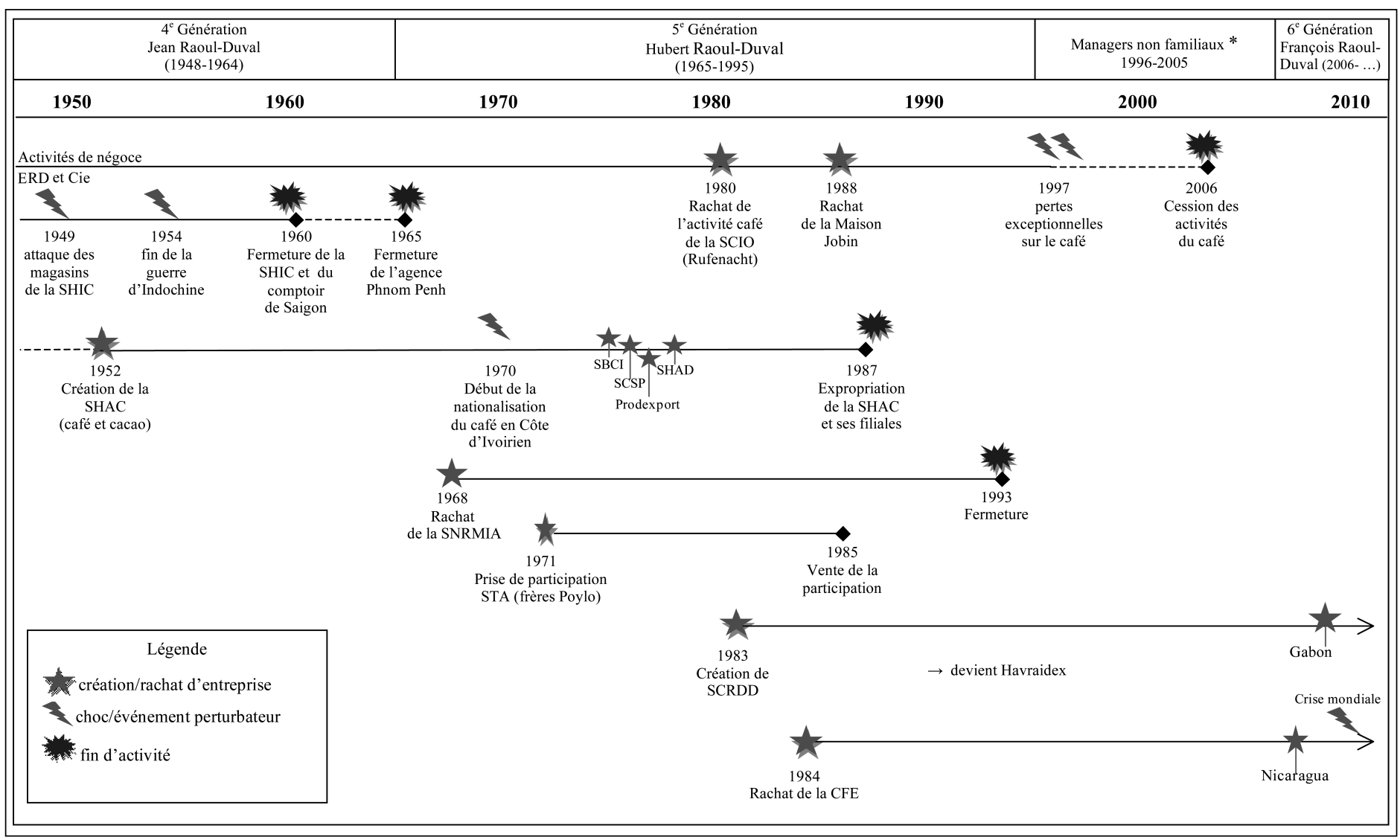

* Les managers non familiaux étaient des personnes de confiance, qui avaient passé une partie de leur carrière dans l'entreprise familiale ou en relation avec elle. 
employant pendant un temps jusqu'à 450 employés, en France et outre-mer.

\section{L'effondrement des cours du café, fin de la niche de spécialisation}

Simultanément à l'aventure de la SHAC en Côte d'Ivoire, ERD et $\mathrm{C}^{\text {ie }}$ continue à faire le négoce du café pour approvisionner les torréfacteurs français, à partir de son siège social havrais. Compte tenu de l'expertise développée dans le café Robusta, les dirigeants décident de renforcer leur position sur le négoce du café en visant des niches de spécialité et en élargissant la gamme des produits proposés. Dans la foulée de la restructuration du marché du café, diverses opportunités se présentent. En 1980, c'est le rachat de l'activité négoce de café de la Société commerciale interocéanique (SCIO) qui permet de diversifier les sources d'approvisionnement et d'ajouter à sa gamme de café l'Arabica en provenance d'Amérique du Sud et d'Amérique centrale, augmentant substantiellement les volumes négociés. En 1988, ce sera le rachat de la très honorable Maison Jobin, fondée en 1871, qui permettra de consolider l'activité café et de contrer en partie l'effet de l'expropriation de Côte d'Ivoire. La maison ERD et $C^{\text {ie }}$ est alors l'un des principaux importateurs français du café.

Mais les mutations structurelles du marché international du café sont beaucoup plus profondes qu'il n'y paraît, les grands groupes agroalimentaires parvenant à se passer des négociants pour leur approvisionnement. Ces derniers perdent du terrain et leurs marges diminuent, d'autant plus que le marché se libéralise et est marqué par l'absence d'entente des pays producteurs à partir de 1989. Reste la spéculation sur les cours, activité à hauts risques qui ne s'ins- crit normalement pas dans le métier de la société ERD et $\mathrm{C}^{\text {ie }}$ mais qui devient incontournable dans les circonstances. Cependant, en 1997, l'entreprise familiale essuie des pertes importantes dans cette activité de trading. Dès lors, l'activité " café " étant désormais perçue comme une activité à la fois fortement cyclique (risquée) et peu rémunératrice, il est décidé de la céder, ce qui sera fait en 2006, après avoir essuyé à nouveau des pertes importantes suite à un retournement des cours du café.

\section{III - DISCUSSION}

Ces éléments permettent de cerner la façon dont ERD et $\mathrm{C}^{\mathrm{ie}}$ a fait preuve de résilience au cours des soixante dernières années: elle a fait face à des chocs importants (guerre et décolonisation, nationalisation et expropriation, pertes exceptionnelles et effondrement de son principal marché) mais à chaque fois, elle a tenté de rebondir dans de nouvelles activités, témoignant des trois dimensions de la résilience.

\section{Absorber les chocs}

La capacité d'absorption des chocs dépend de la surface financière, qu'elle soit effective ou potentielle. Le développement du patrimoine au cours du temps permet de créer un organizational slack, qui peut prendre différentes formes: réserves, immobilisées ou patrimoniales, prises de participation. Renforcé lors des périodes de prospérité, ce patrimoine apporte une garantie dans les situations de crise et, par les revenus qu'il génère, peut permettre d'essuyer les pertes sans pour autant devoir réduire ou cesser l'activité. Cet élément est renforcé par la réputation car, comme le souligne Hubert Raoul-Duval, « ... quand 
on a la chance d'être installé depuis 162 ans (dans la même ville) et de ne pas avoir déposé son bilan, on a un avantage considérable [...] vis-à-vis de ses banquiers: avec les mêmes fonds propres, on a plus de facilités et de confiance qu'on ne l'aurait si nous nous déplacions ailleurs »(1987, p. 11). Mais cela suppose l'adoption d'une ligne de conduite intransigeante en matière de respect des engagements car il y va d'un actif important pour l'entreprise familiale: le « nom de la maison» vaut garantie.

La volonté de continuité est aussi au cœur du cas. Les dirigeants peuvent compter sur les actionnaires familiaux qui leur accordent leur confiance et, au besoin, renoncent à leurs dividendes, preuve d'un capital patient. De fait, si la logique financière est présente, l'affectio societatis - et le sentiment d'appartenance à l'entreprise familiale - motive l'actionnariat familial à préserver la pérennité de la firme. En ce sens, être une entreprise familiale accroît la capacité d'absorption des chocs et favorise la résilience organisationnelle.

\section{Renouvellement et opportunités}

Indéniablement, ERD et $\mathrm{C}^{\text {ie }}$ a fait face à des crises, complètement inattendues au moment des implantations. En ellesmêmes, les activités de négoce outre-mer comportent des risques "normaux » liés aux produits (qualité, disponibilité, accessibilité) et au transport; mais ces risques sont connus et peuvent être gérés (assurances, garanties, etc.). Par contre, les chocs tels que l'expropriation ou la guerre ne sont pas des risques qu'il est possible de gérer avec les instruments habituels. Une stratégie différente doit être mise en place et pour ERD et $\mathrm{C}^{\mathrm{ie}}$, elle a pris le chemin des diversifications tant des sources d'approvisionnement que des produits et des marchés. Ainsi dès les premiers problèmes de la SHIC au Vietnam, les dirigeants amorcent le développement de la SHAC en Afrique, qui est totalement opérante lorsque l'on se retire d'Indochine. Même chose lorsque la Côte d'Ivoire commence son émancipation, une politique de diversification en France est mise de l'avant. Enfin, quand le café décline, d'autres activités ont déjà démarré au sein du groupe familial. Cette capacité de renouvellement prend appui à la fois sur la conception du métier et sur les ressources disponibles au sein de l'entreprise. Elle est aussi le fruit d'une histoire qui se déroule en parallèle d'un métier, le négoce, pour lequel il faut avoir toujours " un coup d'avance » en percevant une opportunité d'affaires là où les autres ne la voient pas. Si la capacité de renouvellement nécessite de faire preuve d'ingéniosité, d'innovation, voire de curiosité, elle demande aussi de disposer d'informations inédites et exactes sur les conditions prévalant sur les marchés locaux ou dans des entreprises susceptibles d'être rachetées. Dans ce domaine, les dirigeants de ERD et $\mathrm{C}^{\mathrm{ie}}$ ont misé sur leur réseau et sur les « hommes de confiance » qui le composent. Souvent des proches, apparentés ou non - avec lesquels sont entretenus des liens forts et des relations de confiance - constitueront des relais et/ou des forces de proposition. Par exemple, le rachat de la CFE se comprend aussi par le fait que, depuis 1949, la famille Westphalen-Lemaître qui en est propriétaire compte toujours un de ses membres au conseil d'administration de ERD et $\mathrm{C}^{\mathrm{ie}}$; les relations d'affaires entre ces deux familles ont donc permis de tisser des liens de confiance qui ont favorisé les tractations. De même, travailler avec un 
partenaire ou prendre une participation dans une affaire se fait intuitu personae: on noue une relation avec une personne qui partage avec nous des valeurs et dont on pense qu'elle nous apportera une compétence tout en étant force de propositions. L'association de ERD et $\mathrm{C}^{\mathrm{ie}}$ avec les frères Poylo, pour créer en 1971 la STA, en est un exemple.

La résilience s'appuie donc sur la combinaison d'une capacité d'absorption des chocs et d'une capacité de renouvellement. On perçoit ici l'importance de cette « orientation entrepreneuriale » de la firme qui permet - à long terme - de survivre aux crises en régénérant les activités de l'entreprise. Sans doute, touche-t-on plus à des valeurs managériales - ou à une culture d'entreprise - qui favorise le renouvellement, qu'à une dimension spécifiquement liée à la famille. Cependant, cette culture de l'EF, qui repose sur une croyance dans l'importance des relations de long terme avec les hommes, correspond à l'accent mis par Miller et Le Breton-Miller (2005) sur la communauté.

\section{La délicate construction de la capacité d'appropriation}

Le fait de traverser des chocs et d'avoir réussi à rebondir signifie-t-il, comme le suggère Cusin (2008), qu'il faut « échouer pour réussir »? Comment les chocs qu'ils ont vécus et surmontés ont-ils contribué à faire évoluer les représentations qu'ont les dirigeants, de leur activité et de la façon de la gérer? Où et comment les expériences sont-elles analysées puis emmagasinées dans la mémoire familiale, pour ensuite en fonder les principes de gestion?

Ainsi que nous l'avons vu, il y a un décalage dans le temps entre le moment où survien- nent les crises et leur éventuelle appropriation. Les apprentissages qui participent à la construction de la capacité de résilience ne se font donc pas de manière immédiate: « la résilience possède une épaisseur temporelle, sans doute nécessaire à son élaboration » (Hollnagel et al., 2009, p. 227). C'est dire que la lecture des procès-verbaux des réunions de $\mathrm{CA}$ correspondant aux trois chocs discutés ne nous dit (presque) rien sur la manière dont ceux-ci ont modifié les représentations des dirigeants de l'époque ou celles des générations futures, quant à ce qu'il convient de faire pour préserver la continuité de l'entreprise. Par contre, les histoires reprises et racontées au fil du temps pour relater la saga familiale, et la connaissance des documents discutés au sein des $\mathrm{CA}$, révèlent que de tels apprentissages ont eu lieu. À titre d'illustration, nous discutons ici de l'évolution du métier, en nous appuyant surtout sur les discussions que nous avons eues avec les dirigeants actuels. Si en 1987, Hubert insistait sur le négoce comme étant le cœur de métier de l'entreprise familiale (cf., note 5), vingt ans plus tard, son fils n'est pas du même avis. Les leçons tirées de l'histoire, avec ses hauts et ses bas, ont amené une révision de ce qu'est le métier de ERD et $\mathrm{C}^{\text {ie }}$ et des compétences uniques qui le fondent. Mais cela ne s'est pas fait sans mal. Il a fallu l'effondrement du marché du café et les pertes énormes qui s'en sont suivies pour que le changement dans les représentations puisse s'opérer donnant lieu à de nombreuses discussions au sein du CA de l'entreprise. Pourtant, il fallait rompre avec les représentations dans lesquelles s'étaient enfermés les dirigeants de la cinquième génération et qui les condamnaient à vouloir à tout prix continuer dans leur métier de 
négociant en commodités qui leur avait apporté de beaux succès financiers. Mais cette conception du métier reposait sur l'intermédiation dans des marchés opaques alors que les changements technologiques et les restructurations ont rendu ces marchés transparents. La venue de la sixième génération couplée aux pertes exceptionnelles qu'a dû essuyer l'entreprise familiale a favorisé une relecture de l'histoire et la redécouverte d'un des principes fondateurs de son succès : rechercher des marges élevées rémunérant la prise de risques sur des marchés où les autres n'osent pas s'aventurer. C'est dire une agilité organisationnelle qui s'apparente au rôle de l'explorateur à la recherche de la terra incognita et qui délimite l'horizon temporel de ses implantations à la période pendant laquelle il est le seul acteur sur le marché (ou en très petit nombre). L'intérêt porté aux extraits végétaux de la CFE se comprend alors car ceux-ci reposent notamment sur une capacité à apporter au client un produit rare, originaire de régions risquées, pour lequel il n'y a pas de substituts et qui permet donc des marges élevées. La réciproque de cette approche du métier demande une forte capacité entrepreneuriale (recherche d'opportunités), des prises de risques mesurées (investissements en partenariat) et une forte diversification des marchés d'approvisionnement sur des niches souvent peu accessibles

La capacité d'appropriation n'est pas à proprement parler spécifique à l'entreprise familiale, bien qu'elle soit ici illustrée par un tel cas. Cependant, si l'apprentissage peut être amorcé par les acteurs peu après les chocs, il peut être poursuivi ultérieurement par d'autres acteurs, voire être complètement décalé dans le temps. Simultané- ment, la capacité d'appropriation met en lumière l'importance de créer un espace réflexif collectif pour que puissent se faire les apprentissages (le CA ici), à défaut de quoi l'entreprise ne pourrait se régénérer en cohérence avec les valeurs de la famille comme de l'entreprise.

\section{CONCLUSION}

Nous l'avons vu, l'histoire du cas ERD et $\mathrm{C}^{\mathrm{ie}}$, c'est l'histoire d'une entreprise qui a surmonté des chocs, déstabilisateurs par nature. Au travers de ses succès et revers de fortune, elle s'est donné les moyens de pouvoir s'inscrire dans la durée au prix de l'abandon de certains produits/marchés et de la réinvention de son métier. Enfin, c'est aussi l'histoire humaine et sociale d'une famille qui a vécu et vit au rythme de l'entreprise.

Au-delà de ce cas, nous avons voulu explorer les implications d'une lecture à travers le prisme de la résilience organisationnelle. Certains événements seront toujours inimaginables et imprévisibles, jusqu'au jour où ils se produisent; c'est ce qu'on appelle des chocs. Explorer les mécanismes et les processus qui permettent à certaines entreprises - familiales ou non - d'y résister et d'en ressortir grandies alors que d'autres s'effondrent et disparaissent est donc une voie de recherche prometteuse. À cet égard, notre travail comporte des limites en ce qu'il ne s'appuie que sur un seul cas. Il convient de poursuivre la perspective par des études portant sur des cas multiples afin de pouvoir comparer des entreprises familiales à d'autres qui ne le sont pas, afin de cerner dans quelle mesure le caractère familial sous-tend vraiment une meilleure capacité de résilience. 


\section{Bibliographie}

Altintas G., Royer I., « Renforcement de la résilience par un apprentissage post-crise: une étude longitudinale sur deux périodes de turbulence »,M@n@gement, vol. 12, n 4, 2009, p. 266-293.

Arregle J-L., Hitt M.A., Sirmon D.G., Very P., "The development of organizational social capital: attributes of family firms", Journal of Management Studies, vol. 44, n 1, 2007, p. 73-95.

Aubourg N., Adaptation ou sélection par l'environnement? Apports et limites de l'écologie des populations à la problématique du changement organisationnel, Thèse de doctorat, Université de Caen, 2000.

Bernard M.J., «La résilience entrepreneuriale les bases d'un dialogue entre deux approches », in R. Zghal, L'entrepreneuriat, Théories, acteurs, méthodes, Sanabil Med, 2007, p. 97-128.

Bonin H., CFAO 1887-2007. La réinvention permanente du commerce outre-mer. Paris, Publications de la SFHOM, 2008.

Christianson M.K., Farkas M.T., Sutcliffe K.M., Weick K.E., "Learning through rare events : significant interruptions at the Baltimore \& Ohio Railroad Museum", Organization Science, vol. 20, n 5, 2009, p. 846-860.

Coutu L.D., "How resilience works", Harvard Business Review, vol. 80, n 5, 2002, p. 46-55.

Cusin J., Faut-il échouer pour réussir?, Paris, Éditions du Palio, 2008.

Cyert R.M., March J.G., A Behavioral Theory of the Firm, Prentice-Hall, 1963.

Danes S.M., Stafford K., Haynes G., Amarapurkar S.S., "Family capital of family firms. Bridging human, social, and financial capital", Family Business Review, vol. 20, n 2 , 2009, p. 1-17.

David A., «Etudes de cas et généralisation scientifique en sciences de gestion », Revue Sciences de Gestion, n ${ }^{\circ}$ 39, 2003, p. 139-166.

De Carolis D.M., Yang Y., Deeds D.L., Nelling E., "Weathering the storm: the benefit of resources to high-technology ventures navigating adverse events", Strategic Entrepreneurship Journal, vol. 3, n 2, 2009, p. 147-160.

Giddens A., La constitution de la société, Paris, PUF, 1987.

Hamel G., Valikangas L., "The quest for resilience", Harvard Business Review, vol. 81, n 9, 2003, p. 52-63.

Hlady-Rispal M., La méthode des cas: application à la recherche en gestion, DeBoeck Université, Bruxelles, 2002.

Hollnagel E., Journé B., Laroche H., «Fiabilité et résilience comme dimensions de la performance organisationnelle: introduction », M@n@gement, vol. 12, n 4, 2009, p. 224-229.

Lengnick-Hall C., Beck T.E., "Adaptive fit versus robust transformation: how organizations respond to environmental change”, Journal of Management, vol. 31, n 5, 2005, p. 738-757. 
Lengnick-Hall C., Beck T.E., "Resilience capacity and strategic agility: prerequisites for thriving in a dynamic environment", Resilience Engineering Perspectives, vol. 2, Preparation and Restoration. Nemeth C.P., Hollnagel E., Dekker S. (Eds), Burlington, VT, Ashgate Publishing Company, 2009.

Malon C., Le Havre colonial de 1880 à 1960, Publications Universitaires, Rouen, 2006.

Miller D., "The correlates of entrepreneurship in three types of firms", Management Science, vol. 29, 1983, p. 770-791.

Miller D., Le Breton-Miller I., Managing for the long run, Harvard Business School Press, 2005.

Smith R., "Mentoring and perpetuating the entrepreneurial spirit within family business by telling contingent stories", New England Journal of Entrepreneurship, October 2009, p. $27-40$.

Somers S., "Measuring resilience potential: an adaptive strategy for organizational crisis planning", Journal of Contingencies and Crisis Management, vol. 17, $\mathrm{n}^{\circ}$ 1, 2009, p. 12-23.

Stevenson H.E., Jarillo J.C., "A paradigm of entrepreneurship: entrepreneurial management", Strategic Management Journal, vol. 11, 1990, p. 17-27.

Stopford J.M., Baden-Fuller C.W., "Creating corporate entrepreneurship", Strategic Management Journal, vol. 15, $\mathrm{n}^{\circ}$ 7, 1994, p. 521-536.

Thorne M., "Interpreting corporate transformation through failure", Management Decision, vol. $38, \mathrm{n}^{\circ}$ 5, 2000, p. 303-314.

Ward J.L., Keeping the family business healthy, San Francisco, CA, Jossey-Bass, 1987.

Weick K.E., "The collapse of sensemaking in organizations: the Mann Gulch Disaster", Administrative Science Quarterly, vol. 38, 1993, p. 628-652.

Weick K.E., Sutcliffe K.M., Managing the Unexpected. Resilient performance in an age of uncertainty, $2^{\text {nd }}$ edition, John Wiley \& Sons, Inc., Hokoben, NJ, 2007.

Yin R.K., Case Study Research, $3^{\text {rd }}$ ed., Sage, Thousand Oaks, CA, 2003. 